\title{
The Red and Infrared IRS WiFS Satellite Data for Mapping of Malaria and JE Vector Mosquito Breeding Habitats \\ Palaniyandi $\mathbf{M}^{*}$ \\ Remote Sensing and GIS Laboratory, Vector Control Research Centre (ICMR), Indira Nagar, Pondicherry-605 006, India
}

The malaria endemic problem is major concern in 14 states and the highland areas of north eastern states and the eight other states of India. The annual prevalence of malaria is estimated 75 million and the death rate is 8 lakhs during the year 1953, and it is gradually reduced to 1.04 million cases and a death rate is 678 in the year 2010 . Though a significant result was obtained by the national malaria control programs, the urban and the highland malaria is a very big challenging problem in India. The Anopheles gambiae are breeding more prolifically in temporary and turbid water bodies, which are ones formed by rain, while in permanent bodies predation becomes important. By contrast, $A$. funestus the principal vectors, which cause malaria. The An. culicifacies-a rural vector, An. stephensi-an urban vector, and An. fluviatilis-a resident of hilly/highland forested areas are causes malaria in most parts of India except at elevations $>1800 \mathrm{~m}$ and in some coastal areas [1-4].

Totally, 152 districts of 20 states in the country were affected with JE epidemics from 1955 to 2012. The epidemics of JE death were occurred mainly in the states of Assam, Andhra Pradesh, Tamil Nadu, Karnataka, Kerala, West Bengal, Goa, Uttar Pradesh, Manipur, Haryana, and Bihar during the period of 2007 to 2012 [5], and the report of cases has been recorded between 1500 and 4000 every year. Japanese encephalitis (JE) is a mosquito borne viral disease with high case fatality with neuropsychiatric disorders. Japanese encephalitis (JE) virus transmitted by the Culex mosquitoes (Culex tritaeniorhynchus, Cx. vishnui, Cx. pseudovishnui, Cx. whitmorei, Cx.epidesmus, Cx.fuscocephala, Cx.gelidus, and Cx. bitaeniorhynchus) JE neurotropic virus predominately affects the thalamus, anterior horns of the spinal cord, cerebral cortex, and cerebellum. The JE is popularly known as "brain fever". It mainly affects children $<15$ years and is mostly asymptomatic [6]. Japanese encephalitis (JE) is a mosquito borne viral disease with high case fatality and grave sequelae with neuropsychiatric disorders. The environmental changes of land use/land cover are, however, most importantly causing the conducing environments than the other variables for survival of JE vector mosquitoes and which are directly influencing the changing nature of epidemiology of the disease in the country [5].

The numbers of traditional, conventional, and modern scientific methods have been using for vector borne disease control, however, the conventional methods are based on the empirical knowledge, it was most conventional and crude methods, laborious, expenditure, erroneous, and time consuming. Therefore, a rapid and advanced technology must be used for the replacement of conventional methods for mapping the problematic areas, and predicting the spatial assessments of disease transmission risk with reliable and high accuracy. Gaining the preeminent understanding of the spatial aspects of vector borne disease transmission, vector ecology, vector breeding habitats, and the occurrences of vector borne disease epidemics in different part of the country, mapping, monitoring and surveillance of the epidemics; towards the achievements of disease transmission control and management, using remote sensing and geographical information systems (GIS) is the most productively significant scientific methods [4-8]. The application of remote sensing and GIS has been significantly developed for the past 25 years for the study of vectors biodiversity, vector abundance [9] and the vector borne diseases with respect to space and time. Remote sensing is a most reliable and repetitive coverage of areal and temporal aspects and it could provide the information on changing the land use/land cover of wetland agricultural practices by introducing the irrigation practices in the newer areas provides the breeding sites for Japanese Encephalitis (JE) and malaria vectors [1013]. The remote sensing of Landsat TM, LANDSAT MSS, IRS LISS I, LISS II, LISS III, IRS CARTOSAT, SPOT, IKONOS, NOVAA-AVHRR etc have been used to analyze the areas suitable for vector breeding habitats, and mapping of vector abundance [1-12].

The present study is to appreciate the use of red and infra-red IRS WiFS multispectral data for land use/land cover mapping on 1:25,000 scale, and the remote sensing resource satellite data (red and infrared/ near infrared spectral data) has been significantly used for mapping the malaria, and JE vector breeding habitats with spatial consistency of overall accuracy of 90 per cent accuracy. The IRS WiFS data with spatial resolution of $188 \mathrm{~m}$ is used for mapping at the state and the national level with 1:250,000 scale. The IRS WiFS spectral signature of near infrared band (NIR) range between $0.77 \mathrm{~nm}-0.86 \mathrm{~nm}$ and red (R) band range between $0.62 \mathrm{~nm}-0.68 \mathrm{~nm}$, is used for deriving the NDVI using the standard formula of $[(\mathrm{NIR}-\mathrm{R}) /(\mathrm{NIR}+\mathrm{R})]$ for mapping the vegetation covers with potential areas of the malaria and JE vector mosquitoes breeding habitats. The calibrated NDVI value has the range between (-) 1.0 and (+) 1.0. The IRS WiFS megabyte quantities of raster data has been integrated with geographical information systems (GIS) to study the landscape environments of surrounding areas of known vector abundance for malaria and JE transmission, as a result, the red and infrared data of the IRS WiFS satellite remote sensing are used for identifying and mapping of potential larval habitats of malaria and JE vectors, and provides the datum of guide lines for assessing the mosquito abundance in the river basins, where the area under wet irrigation rice cultivation was practiced, consequently, mapping of the regions at high, moderate, and low risk of malaria and JE transmission was completed successfully with greatest accuracy and statistically significant.

The Normalized Difference Vegetation Index (NDVI) value between (-)1 and (+)1 derived using the formula of [DN values (Infrared band)-DN values (red band)/DN values (Infrared band) $+\mathrm{DN}$ values (red band)] and the values of the results are recorded

${ }^{*}$ Corresponding author: Palaniyandi M, Remote Sensing and GIS Laboratory, Vector Control Research Centre (ICMR), Indira Nagar, Pondicherry-605 006, India, Tel: 91-143-2274316; E-mail: smp.geog@gmail.com

Received July 04, 2014; Accepted July 09, 2014; Published July 19, 2014

Citation: Palaniyandi M (2014) The Red and Infrared IRS WiFS Satellite Data for Mapping of Malaria and JE Vector Mosquito Breeding Habitats. J Geophys Remote Sensing 3: 126. doi:10.4172/2169-0049.1000126

Copyright: $\odot 2014$ Palaniyandi M. This is an open-access article distributed under the terms of the Creative Commons Attribution License, which permits unrestricted use, distribution, and reproduction in any medium, provided the original author and source are credited. 
excellently within these limits: $0.0-0.2$ corresponds to bare ground, 0.2-0.7 indicates the presence of actively photosynthesizing vegetation, growth stages of vegetation types and the negative values indicate water provides the water depth, colour and chemical compounds of water bodies [9-12]. The maximum breeding and the high abundance of Culex genus JE vector mosquitoes (Cx.tritaeniorhynchus, $C x$. vishnui and $C x$. pseudovishnui, with $C x$. whitmorei, Cx. bitaeniorhynchus) are takes place 4-6-8 weeks after rice transplantation (Figure 1) and the extensive epidemic of encephalitis occurred in most part of Southern India, between the months of August to December [10-13]. The regional scale environmental changes (land use/land cover changes, ecological changes) are the key factors fueled to promoting the malaria and JE and malaria epidemics in newer areas of the country [5-7,10-13]. The intensive rice irrigation cultivation fields supporting the breeding sites are important variable in determining the abundance of mosquitoes associated with the breeding sites and hence, it is sustaining to identify and mapping the malaria and JE vector mosquito larval habitats. The longevity, survival and the high abundance of maximum breeding of malaria vector (Anopheline genus) mosquitoes and JE vector (Culex genus) mosquitoes was found low, moderate, and high in the period of 2-4, 4-6, 6-8 weeks respectively, after wet irrigation cultivation of rice transplantation (Figures 1a-1d), especially in the coastal, river tributary plain and water resource project areas (Figures $4 \mathrm{a}$ and $4 \mathrm{~b}$ ). The regional land use/land cover changes were fuelled to promote the malaria and JE epidemics in newer areas of the country. The coefficient model of malaria and JE vector mosquito abundance are highly correlated with the NDVI, and it is useful in the estimation of mosquito larval abundance and used to predict adult abundance 7-22 days in advance.

The recent years has been witnessed and evidenced by growing incidence of malaria epidemics and JE out breaks in different parts of our country, and more frequently in the districts where the water resource/irrigation projects brought out [5-7,10-13]. These are fueled for mosquito abundance and disease outbreak in and around of the buffer zone of $2.5 \mathrm{Km}$ radius of water projects (irrigation canals, lake,

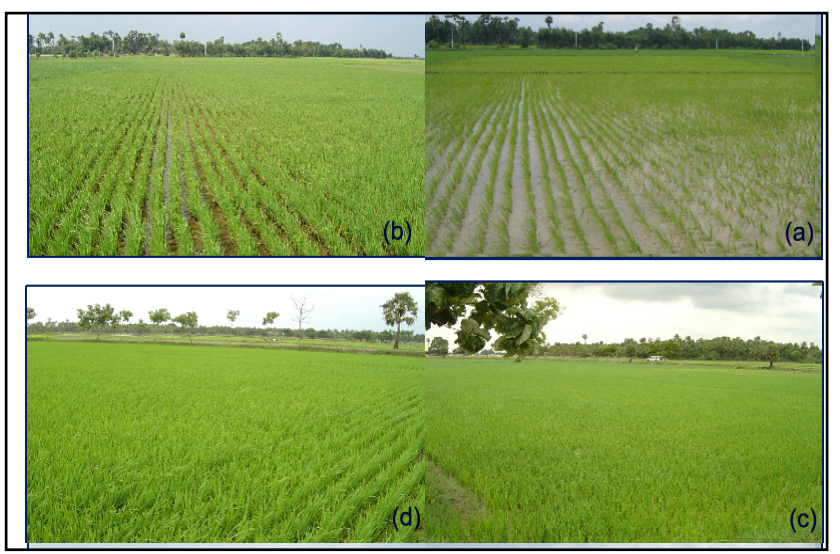

Figure 1: $1 \mathrm{a}=$ Wet irrigation rice cultivation field, 2-4 weeks of rice plantation is the ideal ground for JE vector (Culex groups), and Anophelesgenus malaria immature mosquitoes breeding, beginning considerable breeding

$1 \mathrm{~b}=$ Wet irrigation rice cultivation field, 4-6 weeks of rice plantation is the ideal ground for JE vector (Culexgroups), and Anophelesgenus malaria immature mosquito breedingprofound amount

$1 \mathrm{c}=$ Wet irrigation rice cultivation field, 6-8 weeks of rice plantation is the ideal ground for JE vector (Culexgroups), and Anophelesgenus malaria immature mosquitoes heavy breeding

$1 d=$ Wet irrigation rice cultivation field, $8-10$ weeks of rice plantation is the ideal ground for JE vector (Culexgroups), and Anophelesgenus malaria vectorimmature mosquitoes' moderate to huge breeding. perennial or semi-perennial river/stream, water pools), wetland and cultivation areas (sugarcane, rice and plantain) [10-13]. The type of vegetation cover, vegetation types and growth stage which surrounds the breeding sites (and thereby provides potential resting sites, sugarfeeding supplies for adult mosquitoes and protection from climatic conditions) may play an important role in determining vector abundance may also be important in determining the abundance of mosquitoes associated with the breeding sources [1-12]. The logical step towards the identification and recognition of Japanese Encephalitis (JE) epidemics and the transmission risk is highly associated and witnessed by the incidence of JE spread more frequently in many districts in different parts of the country where the water resource/ irrigation projects was brought out for the intensive wet irrigation rice cultivation agricultural practices, and hence, sea change land use/land cover changes i.e. from dry land to wet land cultivation was practiced throughout the country [4-8]. It is to be noted that the rice irrigation provide breeding sites for Anopheles gambiae early in the growth cycle of the plants, this changes as the rice plants mature and form a dense canopy over the water. The higher NDVI values in the early growing season were found to have higher larval mosquito densities. Importantly, this distinction was possible two months before the peak in larval anopheline and culex numbers.

The remote sensing of JE and malaria epidemics landscape analysis provides the results of vegetation information of NDVI values derived from the image processing of IRS WiFS data, provides the value of $<0.0$ 0.22 corresponds to wet cultivation rice cultivation areas with breeding habitats positives for Culex genus immature JE vector mosquitoes species (Cx. tritaeniorhynchus, Cx. Vishnui, Cx. pseudovishnui, Cx. whitmorei, Cx.epidesmus, Cx.fuscocephala, Cx.gelidus, and Cx. bitaeniorhynchus) positives, and the NDVI value $>0.2$ and $<0.4$ vegetation indicates shows the actively photosynthesizing vegetation, provides guidelines to stratification of areas under vulnerable to the high risk of JE transmission, and followed by the $>0.4$ to $<0.6$ and the $<0.22$ and $>0.13$, having the moderate risk of JE transmission during the Kharif and Rabi crop seasons, and it has been found that 4-6-8 weeks after rice transplantation and which is supporting for the extensive of $\mathrm{JE}$ and malaria epidemic in the newer areas where the wet irrigation rice cultivation is practiced across the country (Figures 1a-1d).

The type of vegetation which surrounds the breeding sites (and thereby provides potential resting sites, sugar-feeding supplies for adult mosquitoes and protection from climatic conditions) may also be important in determining the abundance of mosquitoes associated with the breeding site $[3,4-8,10-13]$. These factors are fueled for malaria vector mosquito abundance and the disease outbreak in and around of the buffer zone of $2.5 \mathrm{Km}$ of water resource projects and wetland cultivation areas [10-13]. The spatial agreement between the observed and predicted values of logistic regression model is $0.757(76 \%)$ sensitivity and specificity of $0.787(79 \%)$ of larval index within a buffer around the trap location of rice fields [10-13]. The Indian Remote Sensing (IRS) satellites Linear Imaging and Self Scanning (LISS) IRS LISSI, LISS-II and LISS-III sensors data has been used for mapping 'mosquitogenic' conditions [4-8]. The coefficient model of rainfall and temperature with the mosquito abundance are highly correlated with the normalized difference vegetation index (NDVI) of NOAA- AVHRR satellite [3-8,10-13], and it is useful in the estimation of mosquito larval abundance and used to predict adult abundance 7-22 days in advance and also estimating Anopheline malaria vector mosquito abundance in the mosquito habitats of rice fields [1-12]. The higher NDVI values (correlated with high rice tiller densities) in the early growing season were found to have higher larval mosquito densities. 
Citation: Palaniyandi M (2014) The Red and Infrared IRS WiFS Satellite Data for Mapping of Malaria and JE Vector Mosquito Breeding Habitats. J Geophys Remote Sensing 3: 126. doi:10.4172/2169-0049.1000126

Page 3 of 4

The recent years has been witnessed and evidenced by growing incidence of vector borne diseases in different parts of our country, and more frequently in the districts where the water resource/irrigation projects brought out (Palaniyandi), and the landscape environment of elevation of less than $220 \mathrm{~m}$ MSL are fueled for mosquito abundance and disease outbreak in and around of the buffer zone of $2.5 \mathrm{Km}$ radius from the water resource projects (Irrigation canals, lake, perennial or Semi-perennial River/stream, water pools), irrigation wet cultivation areas (sugarcane, rice and plantain (Figure 2). The An. culicifacies a rural vector, and An. fluviatilis a resident of hilly-forested areas are causes malaria in most parts of India except at elevations $>1800 \mathrm{~m}$ and in some coastal areas [5-7]. The malaria mosquito habitats, mosquito abundance, parasite incubation, development of parasite, disease transmissions and malaria epidemiology are highly determined by the mosquito ecology, climate change and the environmental (land use/land cover) parameters. Therefore, the survival and longevity of infected mosquitoes and the prevalence of the disease are spatially associated and are definitely controlled by the land use/land cover categories [1-12].

The spatial relationship between land use/land cover changes (dry land to Wetland), the agriculture practice land use categories derived from remote sensing of IRS WiFS data has very good spatial agreement with JE epidemics in different parts of wet cultivation in India produce the results of statistically significant $(\mathrm{r}=0.625, \mathrm{p}$ value $<0.05$ ), it was constructively classified for identifying the JE epidemic risk zones in Karnataka State with $93.4 \%$ accuracy with $100 \%$ specificity (Figure 3) The logical step towards the mapping land use/land cover changes (vegetation changes i.e. deforestation, water bodies, wet lands agricultural practices) guide us for mapping 'mosquitogenic' conditions Importantly, this distinction was possible two months before the peak in larval anopheline numbers.

The occurrence disease epidemics are influenced by several parameters including demography, environmental, geographical and other factors related to vector and parasites. However, climate, landscape, and the environments have been completely controlled the spatial occurrences of horizontal and vertical structure of both vector and disease epidemiology [4-7]. The availability of remote sensing data and GIS are used to mapping land use land cover changes and has been provided the possible information on reliable estimates of breeding habitats and makes reliable estimates of the areas that are

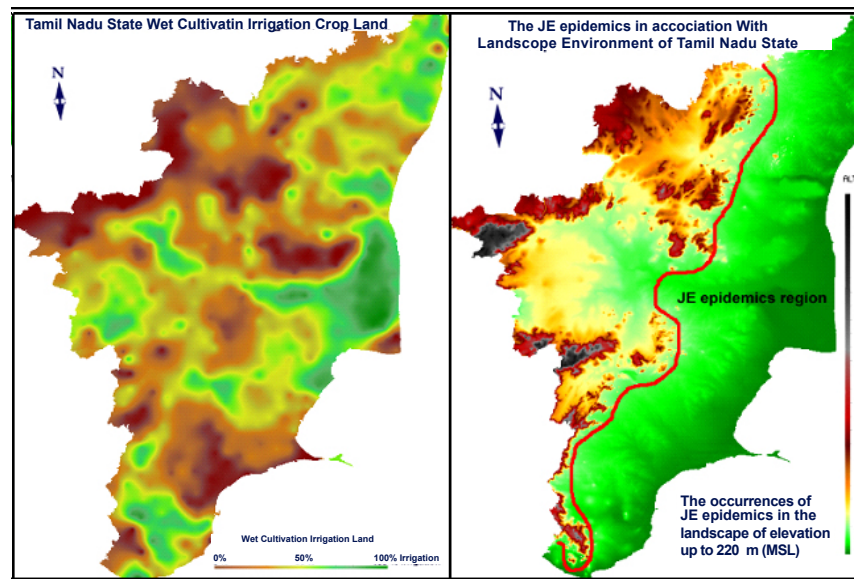

Figure 2: The area under wet cultivation and $<220 \mathrm{~m} \mathrm{MSL}$ is vulnerable to Culexgenus JE vector mosquitoes and Anophelesgenus malaria vector mosquitoesideal breeding ground.

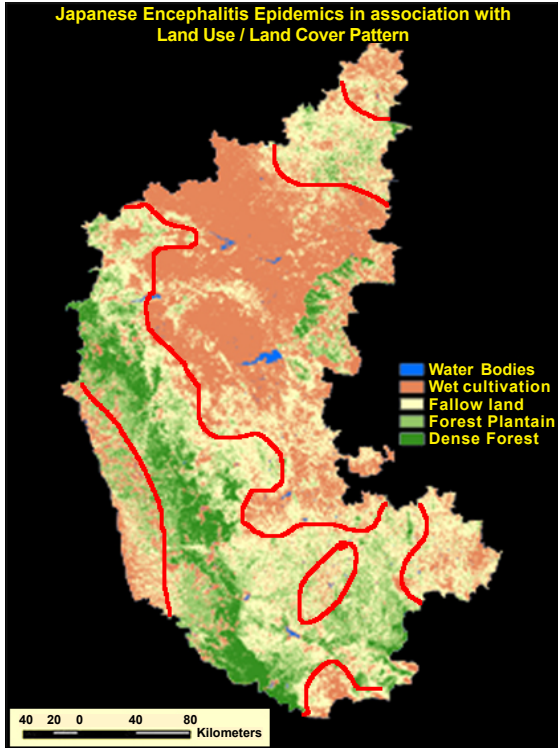

Figure 3: The spatial association between the land use categories (derived from IRS WiFS satellite data) and JE epidemic region in the Karnataka.
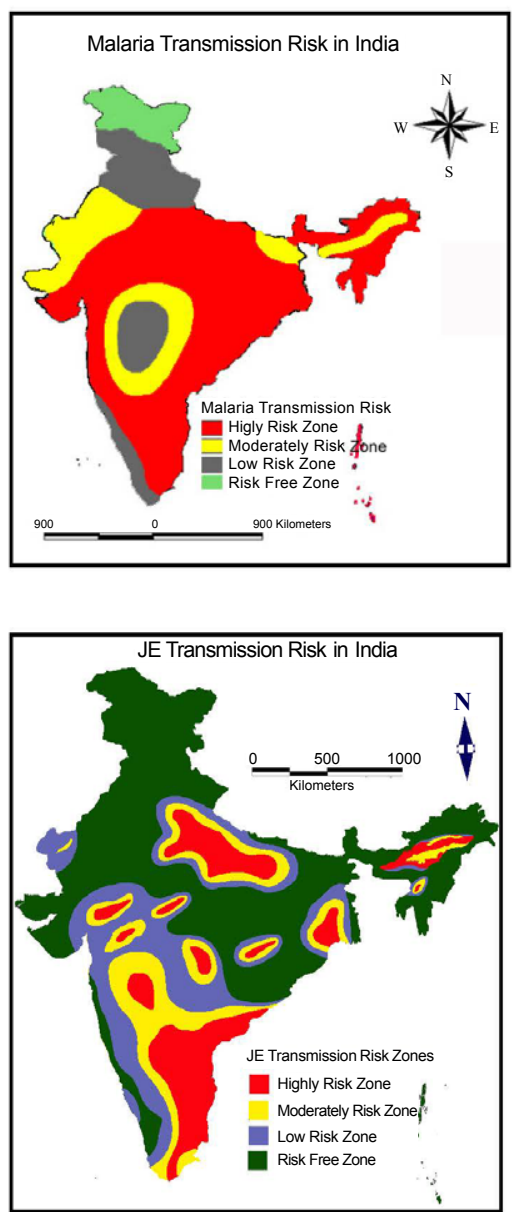

Figure 4: 4a=Malaria Transmission risk in India, based on climate, landscape and the environmental variables [7]; $4 b=J a p a n e s e$ Encephalitis (JE) Transmission risk in India, based on land use /land cover, and the landscape environmental variables. 
Citation: Palaniyandi M (2014) The Red and Infrared IRS WiFS Satellite Data for Mapping of Malaria and JE Vector Mosquito Breeding Habitats. J Geophys Remote Sensing 3: 126. doi:10.4172/2169-0049.1000126

vulnerable to malaria and JE epidemics. The geo-climatic variables are the most important determining key factors that enable to control the vector survival, longevity and disease transmission. The climate factors (temperature, rainfall $(\mathrm{RF})$, relative humidity $(\mathrm{RH})$ and saturation deficiency), are, however, most important determinants than the landscape environmental variables of vector breeding and survival, parasite developments and the spatial diffusion of malaria transmission (Figure 4a). On the other hand, the environmental transmission of land use/land cover categories, and the increase of wet irrigation rice cultivation areas are influenced the spatial spread of malaria and JE transmission in the newer areas (Figure $4 \mathrm{~b}$ ), combined with regional micro climatic changes in the arid and semi-arid areas, and the result has statistically significant with greatest accuracy.

It is concluded that the application of IRS WiFS data is very much useful for mapping land use/land cover categories on 1: 250,000 scale, using maximum likelihood classification, and is to appreciate the red and infrared data for mapping vector breeding habitats of malaria and JE vectors with greatest accuracy. The results explained that the spatial agreements between the malaria and JE transmission risk map and the occurrences of epidemics across the country, respectively, has statistically significant (Figures $4 \mathrm{a}$ and $4 \mathrm{~b}$ ). Therefore, the present study is facilitate to capture the red and infrared data and to integrate with ground truth verification data for mapping of potential vector breeding habitats of both malaria and JE vector mosquito vectors, and thus, provides the guidelines and datum of information for mapping of problematic areas under vulnerable to risk of malaria and JE transmission in the country with greatest accuracy, and thus, choose to appropriate control strategy for vector control at the local and the national, based on the results obtained from the study of remote sensing and GIS [1-12].

\section{References}

1. Ceccato PS, Connor J, Jeanne I, Thomson MC (2005) Application of geographical information systems and remote sensing technologies for assessing and monitoring malaria risk. Parassitologia 47: 81-96.

2. Dale PE, Ritchie SA, Territo BM, Morris CD, Muhar A, et al., (1998) An overview of remote sensing and GIS for surveillance of mosquito vector habitats and risk assessment. J Vector Ecol 23: 54-61.

3. Hassan AN, Onsi HM (2004) Remote sensing as a tool for mapping mosquito breeding habitats and associated health risk to assist control efforts and development plans: a case study in Wadi El Natroun, Egypt. J Egypt Soc Parasit 34: 377-382.

4. Liu J, Chen XP (2005) Relationship of remote sensing normalized differential vegetation index to Anopheles density and malaria incidence rate. Biomed Environ Sci 19: 130-132.

5. Palaniyandi M (2013) GIS for mapping updates of spatial spread and the ecological reasoning of JE transmission in India.

6. Palaniyandi M (2013) GIS mapping of vector breeding habitats. Geospatial World Weekly.

7. Palaniyandi M (2013) Malaria transmission risk in India. Coordinates (GIS e-journal) 9: 42-46.

8. Palaniyandi M (2012) The role of Remote Sensing and GIS for Spatial Prediction of Vector Borne Disease Transmission - A systematic review. Journal of Vector Borne Diseases 49: 197-204

9. Rongnoparut P, Ugsang DM, Baimai V, Honda K, Sithiprasasna R (2005) Use of a remote sensing-based geographic information system in the characterizing spatial patterns for Anopheles minimus $A$ and $C$ breeding habitats in western Thailand. Southeast Asian J Trop Med Public Health 36: 1145-1152.

10. Tucker CJ (1979) Red and photographic infrared linear combinations for monitoring vegetation. Remote Sensing Environ 8: 127-150.

11. Wood BL, Beck LR, Washino RK, Palchick SM, Sebesta PD (1992) Estimating high mosquito-producing rice fields using spectral and spatial data. Int Remote Sensing 13: 2813-2826.

12. Wood BL, Beck LR, Washino RK, Plachick SM, Sebesta D (1991) Spectral and spatial characterization of the rice field mosquito habitat. Int J Remote Sensing 12: 621-626.

13. Wood BL, Washino R, Beck L, Hibbard K, Pitcairn M, et al., (1991) Distinguishing high and low anopheline-producing rice fields using remote sensing and GIS technologies. Prevent Vet Med 11: 277-288. 